\title{
Novel edge states in self-dual gravity
}

\author{
A.P. Balachandran, ${ }^{a}$ Amilcar R. de Queiroz $^{b}$ and M. Arshad Momen ${ }^{c, 1}$ \\ ${ }^{a}$ Physics Department, Syracuse University, \\ Syracuse, New York, U.S.A. \\ ${ }^{b}$ Instituto de Fisica, Universidade de Brasilia, \\ Caixa Postal 04455, 70919-970, Brasilia, DF, Brazil \\ ${ }^{c}$ Department of Physical Sciences, Independent University, \\ Bashundhara R/A, Dhaka-1212, Bangladesh \\ E-mail: balachandran38@gmail.com, amilcarq@unb.br, \\ arshad.momen@gmail.com
}

ABSTRACT: In contrast to the Einstein-Hilbert action, the action for self-dual gravity contains vierbeins. They are eleminated at the level of observables by an $\mathrm{SL}(2, \mathbb{C})$ gauge condition implied by the action. We argue that despite this condition, new "edge" or superselected state vectors corresponding to maps of the spheres $S_{\infty}^{2}$ at infinity to $\operatorname{SL}(2, \mathbb{C})$ arise. They are characterised by new quantum numbers and they lead to mixed states. For black holes, they arise both at the horizon and the spatial infinity and may be relevant for the black hole information paradox. Similar comments can be made about the EinsteinPalatini action which uses vierbeins.

Keywords: Gauge Symmetry, Classical Theories of Gravity

ARXIV EPRINT: 1802.08624

\footnotetext{
${ }^{1}$ On leave of absence from Theoretical Physics Department, University of Dhaka, Dhaka, Bangladesh.
} 


\section{Contents}

1 Introduction 1

2 The Gauss law and edge states in QED 2

2.1 On gauge redundancies in QED 4

3 The self-dual action for gravity 5

4 The self-dual action: edge states $\quad 6$

$\begin{array}{llr}5 & \text { On observables and superselection rules } & 8\end{array}$

$\begin{array}{llr}6 & \text { Mixed states in self-dual gravity? } & 8\end{array}$

\section{Introduction}

There are several systems with more than one Lagrangian for given equations of motion, but when quantised lead to inequivalent quantum systems [1]. They do not differ by total derivatives of functions. We call these Lagrangians as weakly inequivalent Lagrangians. Simple examples of weakly inequivalent Lagrangians on $\mathbb{R}^{N}$ are:

$$
L_{M}=\frac{1}{2} \dot{x}^{i} M_{i j} \dot{x}^{j}, \quad \dot{x}^{i} \equiv \frac{d x^{i}}{d t}, \quad M \in \operatorname{Mat}_{N \times N}(\mathbb{R}), \quad \operatorname{det}(M) \neq 0, \quad M^{T}=M
$$

for different choices of the non-singular symmetric matrix $M$. Here $x=\left(x^{1}, x^{2}, \cdots, x^{N}\right)$ are the coordinates. The matrix $M$ is not varied for finding the equations of motion (1.1). The latter are independent of $M$,

$$
M \frac{d^{2} x^{i}}{d t^{2}}=0 \Rightarrow \frac{d^{2} x^{i}}{d t^{2}}=0
$$

$M$ being non-singular.

For $M=m \mathbb{I}_{N \times N}, m \neq 0, L_{M}$ has the symmetry $x \rightarrow R x, R \in \mathrm{SO}(N)$ but that is not the case for generic $M$. Also the canonical momentum $p_{i}=M_{i j} \dot{x}^{j}$ depends on $M$. For such reasons, quantum theories for different $M$ mutually differ, although that is not the case with the equations of motion.

But there are also familiar Lagrangians in field theories which share the same symmetries and which are regarded as equivalent. Examples are the Einstein-Hilbert, the Einstein-Palatini and the self-dual theory of the Einstein gravity. All of these actions are diffeomorphism invariant, but the last two actions have redundant variables in the form of vierbein variables. The Lagrangian formalism gives rise to first class Gauss law constraints which help us in the elimination of these redundant variables. 
Thus the Gauss law is central to establish some sort of equivalence between these Lagrangians. A naive application of Gauss law does suggest this equivalence. But the Gauss law operator is also a distribution, and therefore must be smeared with test functions. When this aspect is also considered, one finds leftover quantum state vectors associated with spatial infinity carrying new quantum numbers. We have discussed them in various contexts before [2] and we describe them again here, in section 2. In the framework of local quantum physics, they would be associated with superselection rules. We apply the methods described in this section to self-dual gravity and show how edge states on the sphere at spatial infinity, $S_{\infty}^{2}$ arise from the Gauss law.

Similar remarks can be made about the Einstein-Palatini action.

The conclusion is that actions based on vierbeins are not equivalent to the EinsteinHilbert action as they contain additional edge states. The discussion brings out that edge states are to be expected associated with every spatial boundary, such as the black hole horizon $[3,4]$.

Section 3 briefly discusses the known material in the theory of self-dual action. It has a Gauss law based on the non-compact group $\mathrm{SL}(2, \mathbb{C})$. Its edge states, emergent from this Gauss law, are discussed in section 4. In sections 5 and 6 , it is argued that if the observables of this action are to coincide with those of the Einstein-Hilbert action, the above state vectors must lead to mixed states. This is analogous to the results of Balachandran, de Queiroz and Vaidya $[5,6]$ that the colored states in QCD are all mixed.

These remarks are also readily adapted to the Einstein-Palatini action. ${ }^{1}$

\section{The Gauss law and edge states in QED}

Some of the important points of interest in the treatment of Gauss law are already brought out by QED.

At a fixed time, if $E^{i}$ is the electric field and $J_{0}$ the charge density, the Gauss law classically is

$$
\partial_{i} E^{i}(x)+J_{0}(x)=0 .
$$

In quantum theory, following Dirac, (2.1) is regarded as a condition on allowed states vectors $|\cdot\rangle$ :

$$
\left(\partial_{i} E^{i}+J_{0}\right)(x)|\cdot\rangle=0 .
$$

We can regard (2.2) as a condition which picks out the domain of the observables.

But $E^{i}$ in quantum theory are distributions. Thus, we must integrate (2.2) after multiplying by suitable test functions and rewrite it so that derivatives appear only on the test functions. That is because derivatives of distributions are understood dually, in terms of test functions.

So let $C^{\infty}$ denote infinitely differentiable functions, and $C_{0}^{\infty} \subset C^{\infty}$, those functions which also vanish fast at spatial infinity. Then we can rewrite (2.2) as

$$
G(\Lambda)|\cdot\rangle:=\int d^{3} x\left[-E^{i} \partial_{i} \Lambda+\Lambda(x) J_{0}(x)\right]|\cdot\rangle=0,
$$

\footnotetext{
${ }^{1}$ There is recent extensive important work on edge states and low energy theorems by Strominger et al. [7]. We believe that the present work has minimal overlap with theirs.
} 
with $\Lambda \in C_{0}^{\infty}$. Classically, (2.3) implies (2.2) by partial integration since $\Lambda$ vanishes fast at infinity.

The exponentiation of $G(\Lambda)$, namely, $e^{i G(\Lambda)}$, generates a group. The electron field $\Psi_{0}$ under its action acquires a local phase $e^{i \Lambda(x)}$ at $x$,

$$
e^{i G(\Lambda)} \Psi_{0}(x) e^{-i G(\Lambda)}=e^{i \Lambda(x)} \Psi_{0}(x)
$$

and the phase becomes $\mathbb{I}$ at $\infty$, since $\Lambda(x) \rightarrow 0$ as $|\mathbf{x}| \rightarrow \infty$. Thus the group associated with $G(\Lambda)$ can be thought of as the group $\mathcal{G}_{0}^{\infty}$ of maps from $\mathbb{R}^{3}$ to $\mathrm{U}(1)$ which becomes identity at $\infty$, the superscript $\infty$ denoting this fact while the subscript 0 denoting that it is connected to identity.

We note that all "observables" are required to commute with only with these "small" gauge transformations $e^{i G(\Lambda)}$. The electron field $\Psi_{0}$ is not invariant under small gauge transformations. In any case, $\Psi_{0}$ changes sign under $2 \pi$-rotations and thus it is not an observable.

Reverting to (2.3), we can next consider

$$
Q(\mu)=\int d^{3} x\left[-E^{i} \partial_{i} \mu+\mu(x) J_{0}(x)\right]
$$

where $\mu$ approaches a constant $\mu_{0}$ at $\infty$. Let us call the group generated by $\operatorname{such} Q(\mu)$ as $\mathcal{G}_{0}$.

For $\mu \neq \Lambda, Q(\mu)|\cdot\rangle$ need not be zero. If $\mu=\Lambda$, one has $Q(\mu)=G(\mu)$ and thus $Q(\Lambda)|\cdot\rangle=0$. This is the subgroup $\mathcal{G}_{0}^{\infty}=\left\{e^{i Q(\Lambda)}\right\}$ of $\mathcal{G}_{0}$ which acts trivially on $|\cdot\rangle$. Thus the effective group acting on $|\cdot\rangle$ is $\mathcal{G}_{0} / \mathcal{G}_{0}^{\infty}$. The group $\mathcal{G}_{0} / \mathcal{G}_{0}^{\infty}$ is isomorphic to $\mathrm{U}(1)$. For, any element of this quotient is entirely characterized by the value of $\mu$ at spatial infinity.

As an example, choose for $\mu$ a globally constant $\mu=\mu_{0}$. In that case,

$$
Q(\mu)=\mu_{0} \int d^{3} x J_{0}(x)=\mu_{0} Q(\mathbb{I}) \equiv \mu_{0} Q_{0}
$$

where $\mathbb{I}$ is the constant function with the value 1 and $Q_{0}$ is the canonically normalised charge.

An important question now is: how do we create sectors with $Q(\mu) \neq 0$ from the vacuum? For this purpose, we can consider

$$
W(x)=\exp i \int_{x}^{\infty} d x^{\lambda} A_{\lambda}(x)
$$

where the integral is at a fixed time along the spacelike straight line

$$
\{x+\hat{n} l, 0 \leq l<\infty\}
$$

in the direction of a unit vector, say $\hat{n}$. Under a gauge transformation,

$$
A_{\lambda} \rightarrow A_{\lambda}+\partial_{\lambda} \mu
$$

one has

$$
W(x) \rightarrow e^{i \mu_{\infty}(\hat{n})} W(x) e^{-i \mu(x)},
$$


where

$$
\mu_{\infty}(\hat{n})=\lim _{l \rightarrow \infty} \mu(x+\hat{n} l)
$$

Thus, in view of (2.4) and as Dirac observed $[8,9]$,

$$
W(x) \Psi_{0}(x)=\Psi(x)
$$

is invariant under small gauge transformations whereas under large ones,

$$
e^{i Q(\mu)} \Psi(x) e^{-i Q(\mu)}=e^{i \mu_{\infty}(\hat{n})} \Psi(x)
$$

Therefore, if $\mu_{\infty}$ is equal to a constant $\mu_{0}$, the state $\Psi(x)|0\rangle$, where $|0\rangle$ is the vacuum, is the vector with $Q_{0}=1$. But at the same time, it is constant under small gauge transformations.

In $\mathcal{G}_{0}$,we can let $\mu_{\infty}$ be an angle-dependent function. We can generate such elements of $\mathcal{G}_{0}$ by letting $\mu(x)$ approach an angle dependent limit $\mu_{\infty}(\hat{n})$, as indicated by $(2.11)$. Then on exponentiation, and restricting $\hat{n}$ to a Cauchy slice, we get the Sky group of $[10,11]$ isomorphic to the maps from the celestial sphere $S_{\infty}^{2}$ at infinity to $\mathrm{U}(1)$.

As $\mu$ runs over its possibilities, we get a collection of Sky charges $\mu_{\infty}(\hat{n})$ where $\hat{n}$ now fixes the representation of $\mathcal{G}_{0} / \mathcal{G}_{0}^{\infty}$. We have in this case, if $Q(\mu)|\cdot\rangle=0$,

$$
Q(\mu) \Psi(x)|0\rangle=\mu_{\infty}(\hat{n}) \Psi(x)|0\rangle
$$

Since the representation given by (2.14) depends only on the asymptotic data $\mu_{\infty}(\hat{n})$, the corresponding quantum states are edge states.

In the discussion above, we have assumed that the charge of the electron is unity in suitable units. For a field of charge $q, A_{\lambda}$ must multiplied by $q$ while we can also easily restore $q$ appropriately in the above equations. ${ }^{2}$

\subsection{On gauge redundancies in QED}

The fields in the QED Lagrangians are connections $A_{\mu}$ and the electron field $\Psi_{0}(x)$. Gauge invariance implies that there are redundant degrees of freedom in the QED Lagrangian.

The new quantum state vectors which may arise from these redundant degrees of freedom get restricted by the Gauss law. They do not get entirely eliminated however. The charged vectors with charges $q$ and the Sky group vectors characterised by $\mu(\hat{n})$ are examples.

Thus the redundant degrees of freedom in the Lagrangian leave an imprint on the state vectors. It is important that the redundant degrees of freedom implicit in the Lagrangian do not appear at the level of local observables $[2,10,11]$. They must by definition commute with $G(\Lambda)$, but because of the locality, they then commute also with $Q(\mu)$. The reasoning is as follows.

\footnotetext{
${ }^{2}$ In previous work $[12,13]$ (in particular, see the discussion in the appendix), the in-state was constructed using $\hat{n}$ as a time-like unit vector. We can do that here also. But then the commutators of $A_{\lambda}$ with other fields are known only after solving field equations whereas by choosing $x+\hat{n} l$ to lie on a Cauchy slice, we know all the equal time commutators including those of $W$ and $\Psi$. For this reason, in what follows, we choose $\hat{n}$ to be spacelike and be a unit tangent to a spacelike surface.
} 
Let $K$ be a compact spatial region and let $\mathcal{O}_{K}$ be a local observable localised in $K$. Then by (2.3), and its generalisation to local observables

$$
\left[Q(\mu), \mathcal{O}_{K}\right]=\left[Q\left(\left.\mu\right|_{K}\right), \mathcal{O}_{K}\right]
$$

where $\left.\mu\right|_{K}=\mu$ restricted to $K$. But we can find a $\Lambda \in C_{0}^{\infty}$ such that $\left.\Lambda\right|_{K}=\left.\mu\right|_{K}$. In fact there are infinitely many such $\Lambda$. Hence,

$$
\left[Q(\mu), \mathcal{O}_{K}\right]=\left[Q\left(\left.\Lambda\right|_{K}\right), \mathcal{O}_{K}\right]=\left[G\left(\left.\Lambda\right|_{K}\right), \mathcal{O}_{K}\right]=\left[G(\Lambda), \mathcal{O}_{K}\right]
$$

where the last step folows from the fact that the commutator depends only on $\left.\Lambda\right|_{K}$. Hence,

$$
\left[Q(\mu), \mathcal{O}_{K}\right]=0
$$

As the local observables commute with $Q(\mu)$, they are all constructed from electric and magnetic fields. Thus $W(x)$ is not a local observable.

The important result explained in the last few paragraphs is that observables, being local, commute with both small and large gauge transformations.

\section{The self-dual action for gravity}

The material in this section is rather well-known [14]. It is added here for completeness.

The self-dual gravity action in four dimensions is

$$
S=\int d^{4} x(\operatorname{det} e) e_{C}^{a} e_{D}^{b} \mathcal{F}_{a b}^{C D}(A)
$$

where the upper case $C, D \in 1,2,3,4$ are Lorentz group indices and $a, b$ are spacetime indices.

The connection $A$ and its curvature $\mathcal{F}$ are Lorentz Lie algebra-valued, in the self-dual $(1,0)$ representation of the Lie algebra. They are complex. (The antisymmetric product of two four-vector representations contains $(1,0)$ of course).

For the Palatini action in its real form, $A$ and $\mathcal{F}$ are real in (3.1). The connection and the curvature are valued in the four-vector representation of the $\operatorname{SL}(2, \mathbb{C})$ Lie algebra.

As mentioned earlier, (3.1) has redundant degress of freedom associated with $e_{A}^{a}$. This is accompanied by $\mathrm{SL}(2, \mathbb{C})$-gauge invariance.

After Legendre transformation and associated manipulations, one finds the canonically conjugate variables $A_{i}^{C}, \tilde{E}_{D}^{j}[15]$ :

$$
\begin{aligned}
{\left[A_{i}^{C}(x), \tilde{E}_{D}^{j}\right] } & =\delta_{D}^{C} \delta_{i}^{j} \delta^{3}(\mathbf{x}-\mathbf{y}) \\
\tilde{E}_{i}^{A} & =\frac{1}{2} \epsilon^{A B C} \epsilon_{i j k} e_{B}^{j} e_{C}^{k} \Leftrightarrow e_{A}^{i}=\frac{1}{2} \frac{1}{|\operatorname{det} \tilde{E}|^{\frac{1}{2}}} \epsilon_{A B C} \epsilon^{i j k} \tilde{E}_{j}^{B} \tilde{E}_{k}^{C}
\end{aligned}
$$

at a fixed time slice. All other commutators involving $A$ and $\tilde{E}$ vanish on this slice.

Let $T(C)$ be the $\mathrm{SO}(3)$ Lie algebra generators in the spin 1 representation:

$$
T(C)_{A B}=-\epsilon_{C A B}
$$


and set

$$
A_{i}=A_{i}^{C} T(C), \quad \tilde{E}^{j}=\tilde{E}_{D}^{j} T(D) .
$$

Then $\mathcal{F}_{i j}(A)=F_{i j}^{C}(A) T(C)$ is also Lie algebra valued, $F_{i j}^{C}(A) T(C)$ being the curvature of the connection $A_{i}$.

For the self-dual Lagrangian,there are also the following first class constraints in the absence of matter fields:

(i) The Gauss law:

$$
\mathcal{D}_{i} \tilde{E}^{i}|\cdot\rangle=0
$$

on allowed vector state vectors $|\cdot\rangle$. Here we have used a standard notation:

$$
\mathcal{D}_{i} \tilde{E}^{i}=\partial_{i} \tilde{E}^{i}+A_{i}^{\alpha} \tilde{E}^{i \beta}[T(\alpha), T(\beta)] .
$$

(ii) The vector constaint:

$$
\operatorname{Tr} \tilde{E}^{i} F_{i j}|\cdot\rangle=0
$$

(iii) The scalar constraint:

$$
\operatorname{Tr} \tilde{E}^{i} \tilde{E}^{j} F_{i j}|\cdot\rangle=0
$$

\section{The self-dual action: edge states}

We focus on the Gauss law. We must smear it with test functions as before. For this purpose, we let $\Lambda$ and $\mu$ henceforth be Lie algebra valued, $\Lambda \equiv \Lambda^{C} T(C), \mu=\mu^{C} T(C)$ where the functions $\Lambda^{C} \in \mathcal{C}_{0}^{\infty}$ while $\mu^{C} \in C^{\infty}$, i.e. the $\mu^{C}$ can approach angle dependent limits at infinity. Then with

$$
G(\Lambda)=-\int d^{3} x \operatorname{Tr}\left(\mathcal{D}_{i} \Lambda\right) \tilde{E}^{i}
$$

one has

$$
G(\Lambda)|\cdot\rangle=0
$$

which are the Gauss law constraints.

We can also define $Q(\mu)$ :

$$
Q(\mu)=-\int d^{3} x \operatorname{Tr}\left(\mathcal{D}_{i} \mu\right) \tilde{E}^{i}
$$

As mentioned, we now allow the possibility that $\mu^{c}(x)$ approach angle-dependent limits $\mu_{\infty}^{c}(\hat{n})$, as infinity is approached, as in the abelian case.

In what follows, $\mu(\hat{n})$ denotes $\mu^{c}(\hat{n}) T(c)$.

The group that $G(\Lambda)$ 's generate is identified with $\mathcal{G}_{0}^{\infty}$, the group of maps from $\mathbb{R}^{3}$ to complexified $\mathrm{SO}(3)$ which become identity maps at $\infty$ and are also connected to identity. The group that $Q(\mu)$ 's generate is instead $\mathcal{G}_{0}$, which are maps from $\mathbb{R}^{3}$ to complexified $\mathrm{SO}(3)$, whose elements may not approach identity at $\infty$, but are connected to identity. 
If $g$ is an element of $\mathcal{G}_{0}$ or $\mathcal{G}_{0}^{\infty}$, we denote its representative element in quantum theory by $U(g)$.

On quantum states, the effective group is $\mathcal{G}_{0} / \mathcal{G}_{0}^{\infty}$. Its representations give the edge states of the self-dual action. These are edge states since their action on quantum fields only depends on the asymptotic data of $S_{\infty}^{2}$. In particular, in analogy to the U(1) case, if $\mu_{\infty}$ are restricted to constant functions, $Q(\mu)$ generate the complex $\mathrm{SO}(3)$ Lie algebra while if $\mu_{\infty}$ is allowed to have $\hat{n}$-dependence, we get the Sky group or a subgroup thereof. This Sky group is a natural generalisation of the one formulated for gauge theories [2] to the self-dual Palatini action.

To understand this material better, we generalise (2.13) and (2.16). Instead of $\Psi_{0}$ we use $\tilde{E}^{i}$. Then (2.4) becomes

$$
U\left(e^{i \Lambda}\right) \tilde{E}^{i}(x) U\left(e^{-i \Lambda}\right)=e^{i \Lambda(x)} \tilde{E}^{i}(x)
$$

where now $\Lambda(x)$ is a matrix like $T(\alpha)$ while $E^{i}$ is a column vector $\left(E_{C}^{i}\right)$ in the adjoint representation and not its contraction with $T(C)$ as in section 3 . Thus we are changing notation for convenience.

The Wilson line $W(x)$ looks the same as (2.7), except that it now includes a pathordering $\mathcal{P}$ :

$$
\begin{aligned}
W(x) & =\mathcal{P} \exp i \int_{x}^{\infty} d x^{\lambda} A_{\lambda}(x) \\
U\left(e^{i \mu}\right) W(x) U\left(e^{-i \mu}\right) & =e^{i \mu_{\infty}(\hat{n})} W(x) e^{-i \mu(x)}
\end{aligned}
$$

In (2.13), we change $\Psi(x)$ to

$$
W(x) \tilde{E}^{i}(x)
$$

so that

$$
U\left(e^{i \mu}\right) W(x) \tilde{E}^{i}(x) U\left(e^{-i \mu}\right)=e^{i \mu_{\infty}(\hat{n})} W(x) \tilde{E}^{i}(x) .
$$

Note that $\mu_{\infty}(\hat{n})$ is a matrix. We can create vector states characterised by $\mu_{\infty}(\hat{n})$ in its transformation law by starting with a vector $|\cdot\rangle$ invariant under $U\left(e^{i \mu}\right)$ :

$$
U\left(e^{i \mu}\right)|\cdot\rangle=|\cdot\rangle
$$

Then applying (4.7) on the vacuum, we get such a state since one gets

$$
U\left(e^{i \mu}\right) W(x) \tilde{E}^{i}(x)|0\rangle=U\left(e^{i \mu_{\infty}(\hat{n})}\right) W(x) \tilde{E}^{i}(x)|\cdot\rangle .
$$

From this equation, we read off that if $\mu(\hat{n})$ runs over all constant values, we get the complexified $\mathrm{SO}(3)$ whereas if $\mu_{\infty}$ runs over all functions on $S_{\infty}^{2}$, we get the complexified Sky group.

Remarks on unitarity. The transformation property (4.6) of $W(x)$ depends only on $A$ transforming as a connection and not on its reality. Loop quantum gravity program [14] extensively employs this fact. But $W(x)$ is not even formally unitary since $A_{i}$ are complex. The implications of this observation are not clear. 


\section{On observables and superselection rules}

Locality is a concept which is difficult to formulate in quantum gravity, as the concept has to be diffeomorphism invariant. Because of this requirement, no useful diffeomorphism invariant local observables have been found on $\mathbb{R}^{3}$.

Besides this issue, we have the requirement that whatever be the definition of observables, they must commute with $G(\Lambda)$. Now $\tilde{E}_{i}^{a}$ and $e_{a}^{i}$ undergo $\operatorname{SL}(2, \mathbb{C})$-gauge transformations under the action of $U\left(e^{i \Lambda}\right)$. For example,

$$
U\left(e^{i \Lambda}\right) \tilde{E}_{i}^{a}(x) U\left(e^{-i \Lambda}\right)=\left(e^{i \Lambda(x)}\right)^{a}{ }_{b} \tilde{E}_{i}^{b}(x)
$$

where $e^{i \Lambda}$ become identity as $x \rightarrow \infty$. Invariance of observables under (5.1) means that no observable can depend on $\tilde{E}_{i}^{a}$ or $e_{a}^{i}$ for finite $x$ except in $\mathrm{SL}(2, \mathbb{C})$ invariant combinations. But they can depend on the frames "at infinity", the asymptotic forms of $e_{a}^{i}(x)$, as $x \rightarrow \infty$.

In QED, because of locality, local observables commuted with $Q(\mu)$ due to locality [2] since they did so with $G(\Lambda)$. But that argument is not available for the case of gravity.

But suppose that we want the equivalence of the self-dual gravity at the level of observables with the Einstein-Hilbert action. Then we must assume that all its observables $\mathcal{O}$ commute with $Q(\mu)$. To proceed, let us agree on this suggestion. Then if $\mathcal{O}$ is any observable, (4.10) shows that

$$
U\left(e^{i \mu}\right) \mathcal{O} W(x) \tilde{E}^{i}(x)|0\rangle=U\left(e^{i \mu_{\infty}(\hat{n})}\right) \mathcal{O} W(x) \tilde{E}^{i}(x)|\cdot\rangle .
$$

(Here $\mathcal{O}$ is not a matrix while $\tilde{E}^{i}$ is a column vector $\left(\tilde{E}_{b}^{i}\right)$ in the adjoint representation.) It follows that the collection of numbers $\left\{\mu_{\infty}(\hat{n})\right\}$ is characteristic of the representation of the algebra of observables $\mathcal{O}$. It is thus superselected, as $\mathcal{O}$ does not affect this set. [We note that if $U\left(e^{i \mu}\right) \rightarrow U\left(e^{i \mu_{\infty}}\right)$ in (5.2), and similarly $U\left(e^{i \mu^{\prime}}\right) \rightarrow U\left(e^{i \mu_{\infty}^{\prime}}\right)$, then $U\left(e^{i \mu}\right) U\left(e^{i \mu^{\prime}}\right) \rightarrow U\left(e^{i \mu_{\infty}^{\prime}}\right) U\left(e^{i \mu_{\infty}}\right)$, which shows an inversion of the usual ordering. (Just as for $U\left(e^{i \mu}\right), U\left(e^{i \mu_{\infty}}\right)$ and similar expressions represent the quantum operators for their arguments.)]

We can change $\left\{\mu_{\infty}(\hat{n})\right\}$ to $\left\{\mu_{\infty}\left(\hat{n}^{\prime}\right)\right\}$ by changing the direction of the Wilson line and choosing the latter to be

$$
\left\{x+\hat{n}^{\prime} l, \quad 0 \leq l<\infty\right\} .
$$

Since by (5.1), no observable can change $\left\{\mu_{\infty}(\hat{n})\right\}$ to $\left\{\mu_{\infty}\left(\hat{n^{\prime}}\right)\right\}$, they are different representations of $\mathcal{A}$.

Thus we have an infinity of representations characterised by $\hat{n}$, that is, points on the celestial sphere $S_{\infty}^{2}$. They are absent for the observables coming from the Einstein-Hilbert action which does not use the frames.

\section{$6 \quad$ Mixed states in self-dual gravity?}

Let $A_{i}, \tilde{E}^{i}$ represent a particular representation of these fields as conjugate pairs. Then a large gauge transformation $e^{i Q(\mu)}$ transforms them to another equivalent representation (which however may not be unitarily equivalent!), namely $A_{i}, \tilde{E}^{i}$ to $e^{i Q(\mu)} A_{i} e^{-i Q(\mu)}$ and 
$e^{i Q(\mu)} \tilde{E}^{i} e^{-i Q(\mu)}$. Hence also $W$ is changed to $e^{i Q(\mu)} W e^{-i Q(\mu)}$. Thus the new state vector, presumably equivalent in some sense to $W(x) \tilde{E}^{i}(x)|\cdot\rangle$ is,

$$
U\left(e^{i \mu}\right) W(x) \tilde{E}^{i}|\cdot\rangle=e^{i \mu_{\infty}(\hat{n})} W(x) \tilde{E}^{i}(x)|\cdot\rangle
$$

since $|\cdot\rangle$ is invariant by $U\left(e^{i \mu}\right)$ by the assumption in section 5. (Cf. (4.9).)

Observables commute with $U\left(e^{i \mu}\right)$. But still matrix elements of observables between vectors like (6.1) can depend on $U\left(e^{i \mu}\right)$ if it is not unitary. We shall henceforth avoid this problem by considering only $\mathrm{SO}(3)$ - (and not $\mathrm{SL}(2, \mathbb{C})$-) valued gauge transformations. In that case, $U\left(e^{i \mu}\right)$ being unitary, matrix elements of $\mathcal{O}$ between vectors (6.1), are not expected to depend on $e^{i \mu_{\infty}(\hat{n})}$.

By considering $U\left(e^{i \nu}\right) U\left(e^{i \mu}\right)$ etc. in (6.1) where also $e^{i \nu_{\infty}(\hat{n})} \in \mathrm{SO}(3)$, we get a family of vectors in (6.1) giving equal matrix elements for observables $\mathcal{O}$, parametrised by maps of $S_{\infty}^{2}$ to $\mathrm{SO}(3)$.

Consider the density matrices

$$
\rho(\mu)=U\left(e^{i \mu}\right) W_{a}^{b}(x) \tilde{E}_{b}^{i}(x)|\cdot\rangle\langle\cdot|\left(\tilde{E}_{c}^{i}\right)^{\dagger} W_{a}^{-1^{c}}(x) U\left(e^{-i \mu}\right) .
$$

for fixed $a$. By the preceding considerations, for this $\rho$, the expectation value of $\mathcal{O} \in \mathcal{A}$ is independent of $\mu$. That is also the case for convex linear combinations

$$
\sum \lambda_{i} \rho\left(\hat{\mu}^{(i)}\right), \quad \lambda_{i} \geq 0, \sum_{i} \lambda_{i}=1
$$

of such $\rho\left(\hat{\mu}^{(i)}\right)$. That means that $\rho(\hat{\mu})$ is a mixed state, having many decompositions (6.3).

We can generalise (6.3) by replacing $U\left(e^{ \pm i \mu}\right)$ in $(6.2)$ by $U\left(e^{ \pm i \nu}\right) U\left(e^{ \pm i \mu}\right)$. In this way, we get the decomposition of $\rho(\mu)$ as the convex sums of many states.

The vector $|\cdot\rangle$ can be any vector invariant by the action of $U\left(e^{i \Lambda}\right)$, that is, a small gauge transformation. It can in particular be an $\mathrm{SO}(3)$ singlet. Still (6.2) depends on $\mu$ since the remaining factors do not commute with $U\left(e^{i \mu}\right)$. Thus (6.3) depends on $\lambda_{i}$.

We thus find that the above $\mathrm{SO}(3)$ non-singlet states are mixed.

There is a similar result for all $\mathrm{SO}(3)$ non-singlet states. For this purpose, consider a generic $\mathrm{SO}(3)$ non-singlet state $|\psi, \cdot\rangle$ normalised to unity and fulfilling $G(\Lambda)|\psi\rangle=0$. The transformations of $\mathrm{SO}(3)$ are implemented by $U\left(e^{i \mu}\right)$ where $\mu_{\infty}$ is a constant on $S_{\infty}^{2}$. Since $\mathrm{SO}(3)$ is compact, we can reduce its action to a direct sum of irreducible representations. Hence we can write

$$
|\psi\rangle=\sum C_{I I_{3} \lambda}\left|I, I_{3}, \lambda\right\rangle, \quad I \in\{0,1,2, \cdots\}, \quad I_{3} \in[-I,+I] .
$$

where $\lambda$ accounts for labels not covered by $I, I_{3}$. Also $\left|I, I_{3}, \lambda\right\rangle$ are orthonormal.

The observables do not mix $I$ and $I_{3}$ as they commute with $Q(\mu)$. Assume for a moment that they do not mix $\lambda$ as well. Then the density matrix $|\psi\rangle\langle\psi|$ gives the same expectation values as

$$
\sum_{I, I_{3}, \lambda} C_{I I_{3} \lambda}\left|I, I_{3}, \lambda\right\rangle\left\langle I, I_{3}, \lambda\right| \bar{C}_{I I_{3} \lambda}
$$


Each term in (6.5) with $I \neq 0$ leads to a mixed state. That is, the density matrix

$$
\rho_{I I_{3} \lambda}=\left|I, I_{3}, \lambda\right\rangle\left\langle I, I_{3}, \lambda\right|
$$

despite appearance, is mixed $[5,6,12,13]$ ! Thus for example $\operatorname{Tr} \rho_{I I_{3} \lambda} \mathcal{O}=\operatorname{Tr} \rho_{I I_{3}^{\prime} \lambda} \mathcal{O}$, if the two $\rho$ 's are related by an $\mathrm{SO}(3)$-transformation. That is because the latter are generated by $Q(\mu)$ with constant $\mu_{\infty}$ and $Q(\mu)$ commutes with observables by assumption in section 5. More generally, we see that as states on observables, $\rho_{I I_{3} \lambda}$ and $\xi \rho_{I I_{3} \lambda}+(1-\xi) \rho_{I I_{3}^{\prime} \lambda}$ $(0 \leq \xi \leq 1)$ all define the same state (give the same expectation values). Hence (6.5) is mixed.

In general, $\mathcal{O}$ will mix different $\lambda$ 's, but the argument is easily adapted to the case of several $\lambda$ 's in the density matrix (6.5), its term for fixed $I, I_{3}$ then being

$$
\sum_{\lambda, \lambda^{\prime}}\left|I, I_{3}, \lambda\right\rangle C_{I, I_{3}, \lambda} \bar{C}_{I, I_{3}, \lambda^{\prime}}\left\langle I, I_{3}, \lambda^{\prime}\right|
$$

where $C_{I, I_{3}, \lambda}$ are complex numbers. One sees this from (6.4).

The mixed states implied by the existence of the edge states in the self-dual model are quite interesting. They may give clues for the black hole entropy problem.

\section{Acknowledgments}

A.P.B. thanks Rakesh Tibrewala for kindly reminding him about the equation (3.3). We are deeply grateful to Giorgio Immirzi for very helpful suggestions and correcting errors. After submitting this article to the arXiv, our attention was drawn to the reference [16] where edge states in the context of three dimensional gravity is discussed.

Open Access. This article is distributed under the terms of the Creative Commons Attribution License (CC-BY 4.0), which permits any use, distribution and reproduction in any medium, provided the original author(s) and source are credited.

\section{References}

[1] J.F. Carin̂ena, A. Ibort, G. Marmo and G. Morandi, Geometry from Dynamics, Classical and Quantum, Springer (2015).

[2] A.P. Balachandran, T.R. Govindarajan and B. Vijayalakshmi, Particles of Half Integral or Integral Helicity by Quantization of a Nonrelativistic Free Particle and Related Topics, Phys. Rev. D 18 (1978) 1950 [INSPIRE].

[3] A.P. Balachandran, L. Chandar and A. Momen, Edge states in gravity and black hole physics, Nucl. Phys. B 461 (1996) 581 [gr-qc/9412019] [INSPIRE].

[4] A. Momen, Edge dynamics for BF theories and gravity, Phys. Lett. B 394 (1997) 269 [hep-th/9609226] [INSPIRE].

[5] A.P. Balachandran, A.R. de Queiroz and S. Vaidya, Entropy of Quantum States: Ambiguities, Eur. Phys. J. Plus 128 (2013) 112 [arXiv:1212.1239] [InSPIRE]. 
[6] A.P. Balachandran, A.R. de Queiroz and S. Vaidya, Quantum Entropic Ambiguities: Ethylene, Phys. Rev. D 88 (2013) 025001 [arXiv: 1302.4924] [InSPIRE].

[7] A. Strominger, Lectures on the Infrared Structure of Gravity and Gauge Theory, arXiv: 1703.05448 [INSPIRE].

[8] P.A.M. Dirac, Gauge invariant formulation of quantum electrodynamics, Can. J. Phys. 33 (1955) 650 [inSPIRE].

[9] S. Mandelstam, Quantum electrodynamics without potentials, Annals Phys. 19 (1962) 1 [INSPIRE].

[10] A.P. Balachandran and S. Vaidya, Spontaneous Lorentz violation in gauge theories, Eur. Phys. J. Plus 128 (2013) 118.

[11] A.P. Balachandran, S. Kürkçüoğlu, A.R. de Queiroz and S. Vaidya, Spontaneous Lorentz Violation: The Case of Infrared QED, Eur. Phys. J. C 75 (2015) 89 [arXiv:1406.5845] [INSPIRE].

[12] A.P. Balachandran, QCD Breaks Lorentz Invariance and Colour, Mod. Phys. Lett. A 31 (2016) 1650060 [arXiv:1509.05235] [INSPIRE].

[13] A.P. Balachandran and V.P. Nair, An Action for the Infrared Regime of Gauge Theories and the Problem of Color Transformations, arXiv:1804.07214 [INSPIRE].

[14] A. Ashtekar and R. Tate. Lectures on Non-Perturbative Canonical Gravity, World Scientific (1991).

[15] A. Ashtekar, A.P. Balachandran and S. Jo, The CP Problem in Quantum Gravity, Int. J. Mod. Phys. A 4 (1989) 1493 [inSPIRE].

[16] M. Geiller, Lorentz-diffeomorphism edge modes in 3d gravity, JHEP 02 (2018) 029 [arXiv: 1712.05269] [INSPIRE]. 